\title{
Using Process Algebra to Model Radiation Induced Bystander Effects
}

\author{
Rachel Lintott ${ }^{1}$, Celine Addie-Lagorio ${ }^{1}$, and Carron Shankland ${ }^{1}$ \\ Computing Science and Mathematics, University of Stirling, Scotland, FK9 4LA
}

\begin{abstract}
Radiation induced bystander effects are secondary effects caused by the production of chemical signals by cells in response to radiation. We present a Bio-PEPA model which builds on previous modelling work in this field to predict: the surviving fraction of cells in response to radiation, the relative proportion of cell death caused by bystander signalling, the risk of non-lethal damage and the probability of observing bystander signalling for a given dose. This work provides the foundation for modelling bystander effects caused by biologically realistic dose distributions, with implications for cancer therapies.
\end{abstract}

\section{Introduction}

Radiation is often referred to as a double edged sword [11]. Whilst it is one of the most effective treatments for several forms of cancer, exposure to radiation can also be highly carcinogenic. Radiotherapy has been used to treat cancer for over a century, with over half of all modern day patients receiving this treatment at some point [12]. Due to the inherent risks associated with radiotherapy treatments, there is a constant drive to understand the resulting physical and biological processes in order to reduce exposures, both in terms of area exposed, and dose delivered. For many years it has been thought that radiation causes damage to biological cells through radiation tracks traversing the cell nucleus, and damaging the DNA directly. However, over the past 20 years, experimental evidence has been shown to suggest subsequent, non-targeted effects of radiation. It has been suggested recently [2] that these so called 'radiation induced bystander effects' (RIBEs) are mediated through cellular signalling. Cells which have been directly damaged by radiation release signals, in the form of reactive oxygen or nitrogen species. These can be transmitted to surrounding cells either via dispersal into the extracellular medium or via gap-junctions. These signalling molecules are then able induce damage responses in surrounding cells (Figure 1). It has been suggested that RIBEs may be used to amplify the cell killing effect of radiation [19] and hence lead to a reduction in potentially dangerous levels of exposure during treatment. However, RIBEs may also contribute to the increased risk faced by low level exposures to radiation such as those experience during space travel [2]. These effects therefore have a significant impact on our understanding of the biological effects of radiation. Mathematical and computational modelling is therefore essential in understanding the mechanisms at work. 


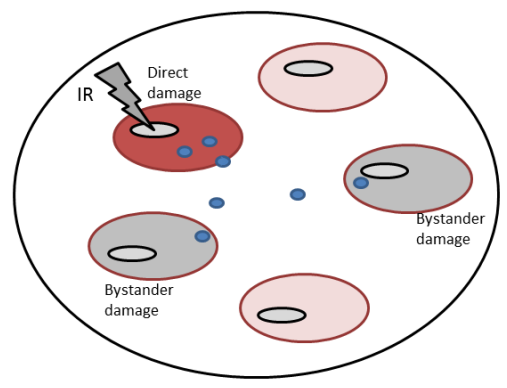

Fig. 1. Basic mechanisms of radiation induced bystander effects. Direct irradation causes damage to the cellular DNA which leads to the production of reactive oxygen or nitrogen species (ROS/RNS). These are released by the cell and can cause damage to neighbouring cells.

In recent years, several mathematical and computational models have been developed to describe this phenomenon $[3,6,7,16]$. Faria and Dickman $[6]$ present an epidemic-type model of the damage caused to a population of cells by the spread of a decaying diffusing signal. Whilst this model has some interesting analytic results, such as the discovery of a critical threshold between the spreading and non-spreading phases of the signal, the assumptions used to develop it are necessarily simplifying, and their paper provides little biological verification. McMahon et al [16], on the other hand, presented a model based largely on the experimental approaches used to assess the impact of bystander effects.

In this paper we present a process algebra model, developed using Bio-PEPA [5], which combines the epidemic-type structure of Faria and Dickman [6] with the biologically verified mechanisms of McMahon [16]. Over recent years, process algebra has been increasingly used in a wide range of biological applications [21, $10,14]$. Bio-PEPA has been specifically developed for use in biological applications, and allows the user to define rates of reaction between distinct species in terms of their concentrations. The underlying continuous time Markov-Chain (CTMC) semantics allows this type of model to be solved in a number of ways, either through stochastic simulation, for example using Gillespie's algorithm, or via conversion of the model to a deterministic system of ordinary differential equations. The level of abstraction afforded by Bio-PEPA is a key attraction to this computational method, allowing biological systems to be defined by a number of species, and the reactions between these species. The versatility in analysis techniques, allowing systems to be solved either deterministically or stochastically, allows Bio-PEPA models to capture properties determined at an individual level, in a computationally less intensive way than more traditionally defined individual based models. The range of applications for which this framework has been used has been expanded from its roots in biochemical networks to areas such as epidemic modelling, crowd dynamics, and population modelling 
of aquatic invertebrates. In this paper, we further extend this range to model cellular damage caused by radiation induced bystander effects.

\section{Model Structure and Assumptions}

The effectiveness of a treatment such as radiation is experimentally tested using a clonogenic survival assay [9], whereby a collection of cells is treated (in this case irradiated) and then split up into individual cells or small clusters of cells and left to grow and divide to form colonies. After a number of days (a week or more) the number of successful colonies (those which have grown to a stated size, usually around 50 cells) is counted and the survival fraction is calculated as

$$
S F=\frac{\text { fraction of successful, treated colonies }}{\text { fraction of successful (untreated) colonies in control }} .
$$

This ratio of treated to untreated colonies accounts for the fact that some colony death may be due to the experimental techniques. Our model has been developed to describe the changes in state of these cell colonies in this experimental set-up.

Modelling in Bio-PEPA requires the definition of three distinct parts; the model compartments, the possible reactions and their rates, and the initial concentrations within each compartment. In this section we describe each of these components, with the Bio-PEPA model in full given in the appendix.

\subsection{Model compartments}

The model presented here has, at its heart, a compartmental, epidemic model structure. Compartmental modelling techniques have been widely used in epidemiology to great effect for many decades [1]. This representation of biological systems has been shown to be appropriate at many, vastly different spatial scales,

from disease control in entire populations or metapopulations [13], to modelling the interactions between viruses and immune cells within hosts [17]. The 'compartments' in these models often refer to infectious classes such as susceptible, infected or recovered, with the rates of transition between classes being determined by the specific biology of the system.

In the context of RIBE, the population we are considering is the fixed number, $N=1000$, of individual cell colonies which have been irradiated. These colonies can be categorised into one of five 'infectious' classes, with $N=H+I+R+A+D$, along with a category tracking the change in signal concentration, as shown in figure 2. Healthy $(\mathrm{H})$ colonies have suffered no damage from either direct effects or bystander signalling. Infectious (I) colonies have been directly irradiated, and as such are able to emit bystander signal (C). Infectious cell colonies that suffer sufficient damage to cause cell death enter the apoptotic class (A). Apoptosis is the method of controlled cell death or suicide. This process leads the cell to break down and disperse in a controlled way, and cells undergoing this process will continue to emit bystander signals. Cells that have undergone apoptosis 


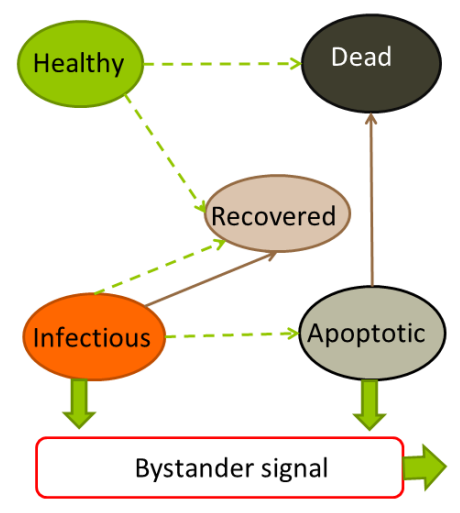

Fig. 2. Schematic showing compartments of the model and transitions between them. Thick arrows show signalling kinetics, dashed arrows show signal mediated transitions, and solid thin arrows show signal independent transitions.

enter the dead (D) class. The inclusion of this class allows the entire population to be a fixed constant, and enables us to easily evaluate the survival fraction as $(N-D) / N$ where $N$ is the total number of cell colonies. The final infectious class consists of those colonies which have suffered some damage but are thought to be relatively stable. These recovered $(\mathrm{R})$ colonies are unable to emit bystander signal, and are not considered to be substantially changed by the presence of signal. The inclusion of this class allows us to acknowledge that some colonies have been affected by treatment with radiation, whilst not being killed, these colonies may have important consequences for subsequent biological responses.

\subsection{Model Reactions}

Cell colonies change state from one compartment to another due to a number of reactions defined in the model. These have been formulated based on the assumptions used by McMahon [16] and are summarised in Table 1. These reactions can be summarised into three parts; bystander signal dynamics, damage suffered as a result of bystander signalling and natural cellular processes.

Bystander signalling dynamics Bystander signal is produced by cells which have suffered some form of direct DNA damage as a result of irradiation. How this direct damage is modelled is discussed in section 2.3. These colonies are those which are initially either infectious or apoptotic. All colonies in these classes emit bystander signal at a rate defined by the reaction emitX (where $\mathrm{X}=\{\mathrm{I}, \mathrm{A}\})$. This rate is equal to $100 \epsilon X / V$ when the concentration of bystander signal is low. As the concentration increases, the rate of emission is limited as the extracellular matrix becomes saturated with signal molecules. The concentration of bystander signal modelled here is not in fact the measurable signal quantity, 


\begin{tabular}{|c|c|c|c|}
\hline Process Label & Description & Definition & Rate \\
\hline damageH & $\begin{array}{c}\text { damage of healthy cells } \\
\text { due to bystander signal }\end{array}$ & $H \rightarrow H-1, R \rightarrow R+1$ & $\rho \beta f(C) H$ \\
\hline deathH & $\begin{array}{c}\text { death of healthy cells } \\
\text { due to bystander signal } \\
\text { damage of infectious cells } \\
\text { due to bystander signal }\end{array}$ & $I \rightarrow I-1, R \rightarrow R+1$ & $\phi \beta f(C) I$ \\
\hline deathI & $\begin{array}{c}\text { death of infectious cells } \\
\text { due to bystander signal }\end{array}$ & $I \rightarrow I-1, A \rightarrow A+1$ & $(1-\phi) \beta f(C) I$ \\
\hline emitI & $\begin{array}{c}\text { emission of bystander signal } \\
\text { by infectious cells }\end{array}$ & $C \rightarrow C+1$ & $\frac{\epsilon I}{V}(100-C)$ \\
\hline emitA & $\begin{array}{c}\text { emission of bystander signal } \\
\text { by apoptotic cells }\end{array}$ & $C \rightarrow C+1$ & $\frac{\epsilon A}{V}(100-C)$ \\
\hline recovery & recovery of infectious cells & $I \rightarrow I-1, R \rightarrow R+1$ & $\gamma I$ \\
\hline apoptosis & death of apoptotic cells & $A \rightarrow A-1, D \rightarrow D+1$ & $\alpha A$ \\
\hline decay & decay of bystander signal & $C \rightarrow C-1$ & $\delta C$ \\
\hline
\end{tabular}

Table 1. Table of model reactions with their corresponding rates

rather the percentage of the saturated quantity. This normalised concentration brings our model in line with that of McMahon, and enables us to use published parameter values. The concentration of bystander signal is reduced through the decay process, which occurs at a rate proportional to the concentration.

It has been suggested in the literature that bystander effects are an 'all or nothing' response [20]. In this case, damage sustained by bystander cells is not dependent on the explicit concentration of signal to which they are exposed, rather damage responses will be triggered at a constant rate if the signal concentration is above a given threshold, $\hat{C}$. McMahon estimates this threshold to be around $21 \%$ of the saturated concentration. In order to capture this 'all or nothing' property, we use the Heaviside step function

$$
\mathbf{h}(C)= \begin{cases}0 & \text { if } \quad C<\hat{C} \\ 1 & \text { if } \quad C \geq \hat{C} .\end{cases}
$$

This on/off switch activates the bystander signal, with no cellular response possible if the concentration is below this threshold.

Damage induced by contact with signal Bystander signal acts only on cell colonies which are healthy $(\mathrm{H})$ or infectious (I). If the concentration of bystander signal is above the specified threshold quantity, then damage occurs to cells at a rate $\beta \mathrm{X}(\mathrm{X}=\{\mathrm{H}, \mathrm{I}\})$. If the signal concentration falls below this threshold quantity, the reaction rate falls to zero, and no further damage is done. Both healthy and infectious cells, when challenged with bystander signal, may either suffer sufficient damage to cause cell death, or may survive this challenge. When a healthy cell colony comes into contact with active bystander signal, a proportion, $\rho$, of these reactions will result in the survival of the colony, and these colonies will move to the recovered class. The rest of the reactions $(1-\rho)$ will result 
in colony death, and the healthy colony will move to the dead class. A similar proportion, $\phi$, of reactions between the infectious colonies and bystander signal, will lead to infectious colony survival, with $(1-\phi)$ of these reactions resulting in colony death.

We wish to relate these proportions $\rho$ and $\phi$ to the damage already suffered by a colony as a result of direct irradiation. To do this, we assume that both direct effects and bystander signal cause a number of 'hits', and, somewhat arbitrarily, that 5 or more hits of damage is sufficient to induce cell death. In line with previous modelling work [16], the number of 'hits' suffered during a challenge with bystander signal is not fixed. Rather, this quantity is poisson distributed, with mean given by the parameter $H_{b}$. This formulation allows us to reduce the number of parameters in the model by collapsing $\rho$ and $\phi$ down to expressions involving the single parameter $H_{b}$. These expressions are given explicitly by

$$
\begin{aligned}
\rho & =\frac{P(1)+P(2)+P(3)+P(4)}{1-P(0)} \\
\phi & =\frac{P(1)(I(1)+I(2)+I(3))+P(2)(I(1)+I(2))+P(3)(I(1))}{(1-P(0)) I_{\text {init }} / N}
\end{aligned}
$$

where $P(i)$ is the probability of receiving $i$ hits from a reaction with bystander signal, and $I(i)$ is the probability of receiving $i$ hits from direct damage.

Natural Cellular Processes The remaining reactions from Table 1 are 'apoptosis' and 'recovery'. These processes are not dependent on the concentration of bystander signal. The reaction 'apoptosis' describes the change of state of a cell colony from apoptotic to dead. The process of apoptosis or cell suicide can take several days to complete, and whilst the colony is clonogenically dead (i.e. unable to continue to grow and produce clones) these cells are still able to emit bystander signal. Without the inclusion of this class, the amount of signal produced, and the duration when the signal is active are underestimated. The reaction 'recovery' enables infectious cells to cease emitting bystander signal. The emission period has been shown to be limited, as damaged cells will begin repair processes. The emission period here is $1 / \gamma$, where $\gamma$ is dependent on the initial dose of radiation delivered. A high initial dose leads to a high $\gamma$, and therefore a slow rate of recovery. Higher doses allow bystander signal to be produced for longer periods by damaged cells.

\subsection{Initial Concentrations}

The final specifications necessary to complete our model are the initial species concentrations. In order to reduce complexity, and to focus our model on the dynamics of bystander effects, we assume that the direct damage due to the initial dose of radiation is fixed. Ionising radiation exposures such as those used in radiotherapy treatments fall into two main categories, electromagnetic (such as $\gamma$ - or X-rays) or particulate (such as $\alpha$ particles). In both cases, damage 
to cells occurs due to the energy deposited within the cell, causing ionisations which can lead to either single or double strand breaks in the DNA. This energy deposition is dependent on the type of radiation to which cells are exposed. In order to provide a generic model of a non-specific radiation treatment, we follow a number of recent models $[15,16,18]$ and describe the damage done by a number of 'hits', $\lambda$, proportional to the radiation dose, $D$, delivered to the cells, hence $\lambda=s D$. If a population of cells is exposed to a uniform dose, then the number of 'hits' suffered by an individual cell is Poisson distributed with mean $\lambda$.

The initial states of the colonies, immediately following irradiation (time $=0)$ is therefore determined as follows

$$
\begin{array}{cc}
H_{\text {init }} & =N e^{-\lambda}, \\
I_{\text {init }}=N \lambda e^{-\lambda}\left(1+\frac{\lambda}{2 !}+\frac{\lambda^{2}}{3 !}+\frac{\lambda^{3}}{4 !}\right), \\
A_{\text {init }} & =N-H_{\text {init }}-I_{\text {init }}, \\
R_{\text {init }} & =0, \\
D_{\text {init }} & =0 .
\end{array}
$$

These states are therefore inherently dependent on the dose delivered.

\subsection{Parameter Estimates}

Since the dynamic behaviour of this model is based on the work of McMahon et. al [16], there is a correspondence between the parameters described, and the relevant estimates. In that paper, the model was parameterised against a number of cell lines, and experiments. Here we focus on the data obtained in [4] for the human prostate cancer cell line DU145. Where there is a correspondence in kinetic rates between our model and McMahons, we use the estimate found in that paper. The exception to this is the parameter $s$ which models the average number of hits per Gy obtained from the direct effect of radiation. Here we set this value to 1 which offers a better fit to the obtainable data than the McMahon estimate $s=0.78$. This parameter is not easily measurable directly, and its closest biological interpretation is the number of unrepaired DNA double strand breaks (DSB). For each Gray, this figure of the order 1 DSB/Gy, and hence $s \approx 1$ is reasonable. The difference in $s$ between our model and that of McMahon is simply down to the structure of the model. McMahon accounts for a heterogeneity in cell response to direct irradiation based on the cell cycle. We avoid this level of complexity by considering a coarser grained, colony level model. This difference in complexity accounts for the slight difference in this parameter estimate.

\section{Results}

The model was simulated using Gillespies stochastic algorithm. For doses between 0 and $8 \mathrm{~Gy}$, at intervals of $0.5 \mathrm{~Gy}, 50$ simulations were run, and the $95 \%$ 


\begin{tabular}{|c|c|c|}
\hline Parameter Name & Symbol & Estimate \\
\hline Hits per Gray & $s$ & $1 \mathrm{hit}$ \\
\hline Rate of bystander damage & $\beta$ & $0.0028 \mathrm{~min}^{-} 1$ \\
\hline Signalling duration & $1 / \gamma$ & $61 /{\mathrm{Gy} \mathrm{min} \mathrm{Gy}^{-} 1}^{-1}$ \\
\hline Rate of signal emission (at low concentration) & $\epsilon$ & $0.00011 \mathrm{~min}^{-} 1$ \\
\hline Rate of signal decay & $\delta$ & $0.019 \mathrm{~min}^{-} 1$ \\
\hline Hits per interaction with bystander & $H_{b}$ & $3.9 \mathrm{hits}^{-1}$ \\
\hline Rate of death due to apoptosis & $\alpha$ & $0.000417 \mathrm{~min}^{-} 1$ \\
\hline
\end{tabular}

confidence intervals on the survival fraction were calculated. From these experiments, we found that the largest confidence interval of $0.95 \pm 0.0058$ was found at a dose of $1 \mathrm{~Gy}$. This is equivalent to a confidence interval of around $0.1 \%$ of the mean. Since the variation in simulation outputs is so low, the mean output of the simulations is used.

In order to validate the model, the output was compared to survival data from experiments published in [4]. This data is in the form of the surviving fraction of cell colonies, taken several days after treatment with ionising radiation. In order to simulate this, we ran the model for 10000 time steps, simulating approximately 7 days. By this point, the system had reached equilibrium, and simulation over longer periods provided negligibly different results. The survival fraction predicted by the model shows a good fit to the available data, with the predicted output falling within experimental confidence limits (Figure 3).

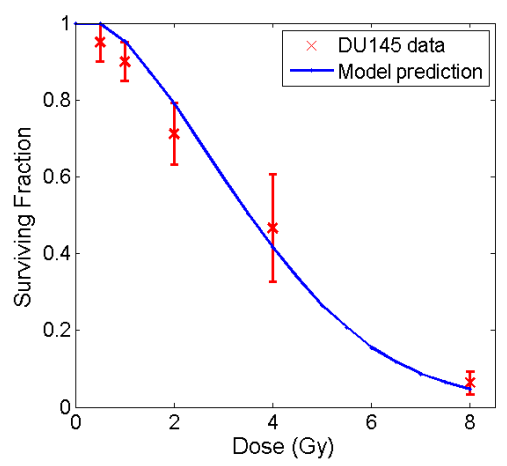

Fig. 3. Survival curve predicted by model and compared with data from Butterworth et al [4]. Model has been parameterised using McMahon [16] for the human prostate cancer cell line DU145.

Having validated the model, against experimental data, we are able to produce a number of predictive results. Figure 4 (a) shows the proportion of cell colonies killed by either direct effects of irradiation or by bystander effect. It is clear from this plot that for high doses, the proportion of cells killed by bystander 
signalling is small when compared to direct effects. Doses up to around 3 Gy see a higher proportion of colony death being attributed to bystander effects than the direct effect of irradiation, hence it is in this range of exposures that bystander effects are most important. This result is significant since it is this range of dosages that is used in the clinical setting. High exposures of radiotherapy treatments are often delivered as fractionated doses with a typical fraction being around 2Gy. This same range of doses predicts the peak levels in the number of
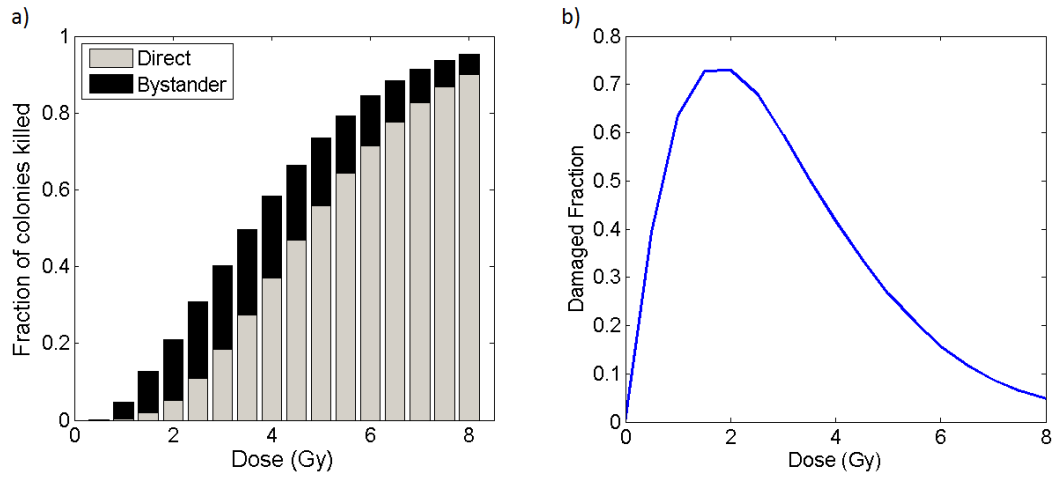

Fig. 4. Left hand plot: Proportion of colonies killed through direct effects and bystander signalling. Right hand plot: Recovered fraction of cell colonies. A peak in recovered colonies is observed at low initial doses.

cell colonies which are assumed to be 'recovered' (Figure 4 (b)). These colonies have been damaged at low levels but maintain the ability to divide. This class of cells is particularly important when considering the negative knock-on effects of radiotherapy, since these cells are more prone to detrimental mutations, possibly leading to long term problems for the health and well-being of the patient.

The Bio-PEPA Java plug-in allows direct calculation of probability and cumulative distribution functions for a specified target output. Due to the on/off switch in signal activity present in this model, these functions allow us to quantify the risk of seeing an actively damaging bystander effect for a given initial dose. By specifying the target output as $\mathbf{h}(C)=1$, the PDF output gives the time at which each simulation reached this condition. Once more, we consider the range 0-8Gy, at intervals of 0.5 Gy. For each dose considered, 1000 simulations were run for a length of 10000 time steps (minutes). The number of simulations performed in this case has been increased to allow greater accuracy, for each dose the time taken to simulate the model 1000 times was of the order of around 1 second. The time to bystander onset was calculated for each simulation, along with the proportion of all simulations where bystander effect was observed. The median time to bystander onset, along with $25 \%$ and $75 \%$ quartiles are shown in Figure 5. For the lowest dose considered (0.5Gy), only $0.6 \%$ of all simulations predicted a bystander response. At this dosage, the risk 
of producing enough bystander signal to cause damage or death to surrounding cells is therefore very low. At doses above 1.5Gy however, all simulations predicted a bystander response.. The median time between direct irradiation and bystander onset reduces with increasing dose, before saturating at a lower limit of around 12-13 minutes.
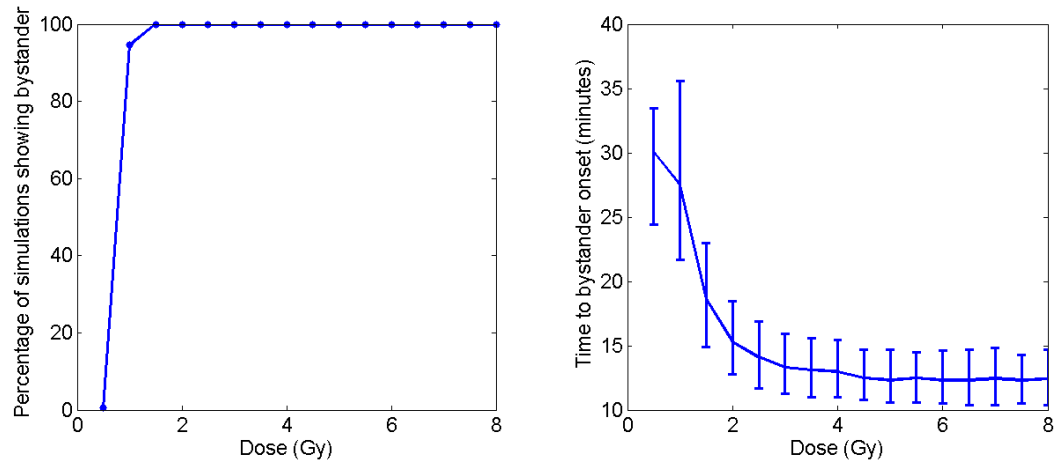

Fig. 5. Left plot shows the percentage of simulations resulting in the onset of bystander effect. Right plot shows median time to bystander onset, with error bars showing upper and lower quartiles

\section{Discussion}

In this paper we have presented a model of the in vitro consequences of radiation induced bystander effects. By basing our model structure on that of an epidemic type model, considering the transitions between 'infectious' classes, in combination with the biologically relevant assumptions of previous modelling work [16], we have been able to identify a number of key results. We have shown that the dose region up to around $3 \mathrm{~Gy}$ presents a significant risk of bystander effect, both in terms of the proportion of death attributed to these effects (Figure 4) and the number of stable cell colonies suffering minor damage (Figure 4(b)). By developing this model in Bio-PEPA we have easily been able to extract information about the risk of observing bystander effect for a given dose, given that emission of these signals is inherently a stochastic process. Along with an evaluation of the risk of bystander signalling, we have predicted the time between direct irradiation and bystander onset for a range of doses, showing that this time lag reaches at a minimum of around 12 minutes for doses above around $2 \mathrm{~Gy}$.

The discovery of radiation induced bystander effects, in both the in vitro and in vivo settings, has important consequences for the future of radiation research. These effects must be taken into account when evaluating the risk of low level background exposures, and also when developing radiotherapeutic 
strategies for the treatment of cancer. Delivery of radiation doses and their effects can no longer be thought of as targeted point in time damage to specific cells. The number of cells killed by a dose of radiation has been modelled effectively for many years, primarily through the linear-quadratic model (LQ), and the biologically effective dose (BED) [8]. The LQ model offers a prediction of the fraction of cell kill for a single given dose of irradiation, whilst the BED converts the total dose delivered over a number of fractions (typically of around $2 \mathrm{~Gy}$ ) given at discrete times into the single dose required to achieve the equivalent cell kill. Whilst this method of modelling cell death has been clinically effective, this approach neglects the non-lethal effects of bystander signalling. The model presented here shows a peak in the risk of bystander effects, leading to both cell death and damage, at around 2-3Gy. This result has been observed both in previous modelling work (ref) as well as experimentation (ref).

Our prediction that the number of 'recovered' cell colonies peaks at clinically relevant doses suggests that dose fractionation may increase the risk of side effects due to bystander damage. These side effects may include long term mutations, leading, for example, to the development of tumours. The model presented here takes into account both the lethal and non-lethal effects of bystander signalling, this key property is novel to this approach, and has been facilitated by the use of Bio-PEPA in this modelling work.

The model presented here is a step in investigating of the impacts of bystander effects in clinical situations. To develop this work further, an in-depth analysis of the parameter estimates should be done with further experimentation done to validate our predictions, in particular those shown in Figure 5. In order to estimate experimentally unobtainable parameters, modelling in Bio-PEPA facilitates the use of evolutionary computation tools for model fitting [14].

\section{Acknowledgements}

We acknowledge the input of both Stephen McMahon and Kevin Prise, (Queens University, Belfast) in the development of this model and the radiobiological interpretation of the results. R. Lintott and C. Shankland are funded by the EPSRC project EP/K039342/1. C. Addie-Lagorio was funded by the Wellcome Trust Biomedical Vacation Scholarship.

\section{References}

1. Anderson, R.M., May, R.M.: Infectious diseases of humans, vol. 1. Oxford university press Oxford (1991)

2. Azzam, E.I., de Toledo, S.M., Harris, A.L., Ivanov, V., Zhou, H., Amundson, S.A., Lieberman, H.B., Hei, T.K.: The ionizing radiation-induced bystander effect: evidence, mechanism, and significance. In: Pathobiology of Cancer Regimen-Related Toxicities, pp. 35-61. Springer (2013)

3. Brenner, D., Little, J., Sachs, R.: The bystander effect in radiation oncogenesis: Ii. a quantitative model. Radiation research 155(3), 402-408 (2001) 
4. Butterworth, K.T., McGarry, C.K., Trainor, C., O'Sullivan, J.M., Hounsell, A.R., Prise, K.M.: Out-of-field cell survival following exposure to intensity-modulated radiation fields. International Journal of Radiation Oncology Biology Physics 79(5), 1516-1522 (2011)

5. Ciocchetta, F., Hillston, J.: Bio-pepa: A framework for the modelling and analysis of biological systems. Theoretical Computer Science 410(33), 3065-3084 (2009)

6. Faria, F.P., Dickman, R.: An epidemic process mediated by a decaying diffusing signal. Journal of Statistical Mechanics: Theory and Experiment 2012(06), P06006 (2012)

7. Faria, F.P., Dickman, R., Moreira, C.H.: Models of the radiation-induced bystander effect. International journal of radiation biology 88(8), 592-599 (2012)

8. FOWLER, J.: 21 years of biologically effective dose. The British Journal of Radiology 83, 554-568 (2010)

9. Franken, N.A., Rodermond, H.M., Stap, J., Haveman, J., Van Bree, C.: Clonogenic assay of cells in vitro. Nature protocols 1(5), 2315-2319 (2006)

10. Guerriero, M.L., Pokhilko, A., Fernández, A.P., Halliday, K.J., Millar, A.J., Hillston, J.: Stochastic properties of the plant circadian clock. Journal of The Royal Society Interface p. rsif20110378 (2011)

11. Hei, T.K., Zhou, H., Ivanov, V.N., Hong, M., Lieberman, H.B., Brenner, D.J., Amundson, S.A., Geard, C.R.: Mechanism of radiation-induced bystander effects: a unifying model. Journal of Pharmacy and Pharmacology 60(8), 943-950 (2008)

12. Joiner, M.C., van der Kogel, A.: Basic Clinical Radiobiology Fourth Edition. CRC Press (2009)

13. Lintott, R.A., Norman, R., Hoyle, A.: The impact of increased dispersal in response to disease control in patchy environments. Journal of theoretical biology 323, 57-68 (2013)

14. Marco, D., Scott, E., Cairns, D., Graham, A., Allen, J., Mahajan, S., Shankland, C.: Investigating co-infection dynamics through evolution of bio-pepa model parameters: a combined process algebra and evolutionary computing approach. In: Computational Methods in Systems Biology. pp. 227-246. Springer (2012)

15. McMahon, S.J., Butterworth, K.T., McGarry, C.K., Trainor, C., O'Sullivan, J.M., Hounsell, A.R., Prise, K.M.: A computational model of cellular response to modulated radiation fields. International Journal of Radiation Oncology* Biology* Physics 84(1), 250-256 (2012)

16. McMahon, S.J., Butterworth, K.T., Trainor, C., McGarry, C.K., O?Sullivan, J.M., Schettino, G., Hounsell, A.R., Prise, K.M.: A kinetic-based model of radiationinduced intercellular signalling. PloS one 8(1), e54526 (2013)

17. Nowak, M., May, R.M.: Virus Dynamics: Mathematical Principles of Immunology and Virology: Mathematical Principles of Immunology and Virology. Oxford university press (2000)

18. Partridge, M.: A radiation damage repair model for normal tissues. Physics in medicine and biology 53(13), 3595 (2008)

19. Prise, K.M., O'Sullivan, J.M.: Radiation-induced bystander signalling in cancer therapy. Nature Reviews Cancer 9(5), 351-360 (2009)

20. Schettino, G., Folkard, M., Michael, B.D., Prise, K.M.: Low-dose binary behavior of bystander cell killing after microbeam irradiation of a single cell with focused ck x rays. Radiation research 163(3), 332-336 (2005)

21. Scott, E., Hoyle, A., Shankland, C.: Pepa'd oysters: Converting dynamic energy budget models to bio-pepa, illustrated by a pacific oyster case study. Electronic Notes in Theoretical Computer Science 296, 211-228 (2013) 